\title{
Experiencia en loxoscelismo cutáneo y cutáneo visceral de manejo hospitalario: clínica, evolución y propuesta terapéutica
}

\author{
Constanza del Puerto, Cristóbal Saldías-Fuentes, Maximiliano Curi, \\ Camila Downey y Romina Andino-Navarrete
}

Pontificia Universidad Católica de Chile, Santiago, Chile Departamento de Dermatología

(CDP, CSF, MC, CD, RAN)

Establecimiento donde se efectuará el estudio: Hospita Clínico Universidad Católica de

Los autores declaran la ausencia de conflictos de interés.

No hubo fuentes de financiamiento.

Recibido: 15 de septiembre de 2017

Aceptado: 10 de abril de 2018 .

Correspondencia a: Constanza del Puerto Troncoso mcdelpue@uc.c

\section{Experience in visceral cutaneous and cutaneous loxoscelism of hospital management: clinical, evolution and therapeutic proposal}

Background: Loxoscelism is a common pathology in our environment with a broad spectrum of differential diagnoses and presentations, with potentially serious complications, even to the point of death. To date, there is no standard treatment for these patients. Aim: To describe the clinical manifestations, main complications, therapeutic management, and evolution of loxoscelism in an inpatient setting from a tertiary hospital in Chile. Methods: All patients consulting and hospitalized in the hospital of the Pontificia Universidad Católica de Chile with diagnosis of loxoscelism between 2014 to 2017 and evaluated by dermatologist were included. Review of clinical files, including symptoms, images, laboratory parameters and treatment. Results: We evaluated seventeen inpatient with loxoscelism, whose presentation responds to the national epidemiological pattern. Most cases were managed with antibiotics, systemic corticosteroids, antihistamines, and dapsone. From these, $11.8 \%$ corresponded to viscerocutaneous loxoscelism, successfully managed with supportive measures, systemic corticosteroids and antihistamines. Fifty-nine percent healed their cutaneous lesions after one month of treatment, with slight residual scarring or post inflammatory hyperpigmentation, without associated mortality in our series. Discussion: Most cases of cutaneous loxoscelism presented excellent response and rapid resolution of the disease after combined therapy with systemic corticosteroids, antibiotics and dapsone, suggesting that the use of these therapies could stop the progression of cutaneous necrosis and prevent complications associated with loxoscelism.

Key words: Loxoscelism; dapsone; Loxosceles laeta.

Palabras clave: Loxoscelismo; dapsona; Loxosceles laeta.

\section{Introducción}

E 1 loxoscelismo es el cuadro clínico producido por la mordedura de la araña del género Loxosceles. Existen más de 100 especies de arañas de este género distribuidas en el mundo, presentándose la mayoría en Sudamérica ${ }^{1}$. La principal especie en Chile es Loxosceles laeta, conocida también como la "araña de rincón”, presente desde la Región de Antofagasta (II ${ }^{\circ}$ Región) hasta la Región del Bío Bío (VIII ${ }^{\circ}$ Región), aunque se han descrito casos a lo largo de todo el país ${ }^{2}$. En Chile, se estima que esta araña se encuentra presente en nueve de cada 10 hogares $^{3}$, registrándose 450 casos de loxoscelismo al año, con una mortalidad de hasta cuatro casos por año ${ }^{4}$

Las arañas Loxosceles se caracterizan por tener actividad nocturna ${ }^{1}$, prefiriendo ambientes cálidos y alejados del sol, pudiéndose encontrar en clósets, rincones de habitaciones, detrás de cuadros y en cualquier otro lugar oscuro y seco. Son de comportamiento no agresivo, por lo que su mordedura representa un mecanismo de defensa ${ }^{1}$. Mide entre 10 y $15 \mathrm{~mm}$, y con sus patas extendidas, entre 25 a $42 \mathrm{~mm}$. Presenta características físicas distintivas, con un cuerpo en forma de violín, de color café más oscuro en el abdomen, y la presencia de tres pares de ojos distribuidos en forma semicircular ${ }^{5}$.

La mayoría de las mordeduras ocurre en el período entre primavera y verano ${ }^{6}$, en la noche o en la madrugada ${ }^{7}$. Las zonas del cuerpo más frecuentemente afectadas son el muslo, el tronco y la zona proximal de los brazos ${ }^{1}$. El loxoscelismo se presenta principalmente en mujeres, en zonas urbanas y urbano-marginales y dentro del hogar ${ }^{8}$.

El veneno de esta araña es dermo-necrótico y víscerotóxico ${ }^{9,10}$. El principal componente tóxico es la esfingomielinasa $\mathrm{D}$, que interactúa con las membranas celulares y otros elementos tisulares desencadenando alteraciones que involucran el sistema del complemento y activación de neutrófilos ${ }^{11}$. 
Se han descrito dos cuadros clínicos característicos producto de la mordedura de esta araña: el loxoscelismo cutáneo (LC) que representa $81,2 \%$ de las manifestaciones, y el loxoscelismo cutáneo visceral (LCV) que representa el 18,8\% restante ${ }^{7}$. Dentro de las presentaciones de LC se ha descrito una presentación infrecuente ( $4 \%$ de los casos) conocido como loxoscelismo cutáneo predominantemente edematoso $(\mathrm{LCPE})^{12}$. Este último se presenta en casos en que la mordedura ocurre en la cara, manifestándose con edema, sin isquemia ni placa livedoide (probablemente ya que el edema genera dilución del veneno en los tejidos), siendo en general de buen pronóstico, con recuperación en una semana y con bajo riesgo de generar $\mathrm{LCV}^{13}$.

El LC es generalmente benigno, siendo las manifestaciones más frecuentes el dolor, edema y eritema ${ }^{1}$. Con respecto al cuadro clínico se describe un dolor tipo urente en 91 a $100 \%$ de los $\operatorname{casos}^{14}$, que puede aparecer desde el momento de la mordedura o tener una latencia variable entre cinco minutos y cuatro horas. En general, se acompaña de hiperestesia perilesional, con edema duro o elástico que aparece en las primeras horas ${ }^{7}$. En seis a ocho horas progresa a un halo de vasoconstricción e isquemia que se extiende alrededor de la lesión ${ }^{15}$, pudiéndose formar después una ampolla en alrededor de 50\% de los casos, generalmente sin adenopatías regionales ${ }^{7}$. La presentación típica de la placa livedoide se establece a las $24 \mathrm{~h}$ como una placa violácea central, halo pálido y borde eritematoso ${ }^{7}$. La zona isquémica central puede evolucionar a necrosis en tres a cuatro días y formar una escara en cuatro a siete días que luego se desprende dando paso a una úlcera ${ }^{16}$. La mordedura tiende a curar por segunda intención en seis a ocho semanas ${ }^{7,17}$.

El LCV ocurre si el veneno alcanza el torrente sanguíneo, desencadenando sus efectos necrosantes, hemolíticos, vasculíticos y coagulantes. Los pacientes comienzan con fiebre, compromiso del estado general, hemoglobinuria y hematuria, evolucionando con ictericia y compromiso de conciencia. La hemoglobinuria y hematuria están presentes en $100 \%$ de los LCV. En la mayoría de los casos, la fiebre, ictericia o hematuria se presentan desde las primeras seis horas de evolución ${ }^{7}$.

El loxoscelismo tiene una letalidad general de 3\%, y llega a ser de 20 a $25 \%$ en los cuadros de $\mathrm{LCV}^{16}$. La letalidad depende principalmente de la latencia en el inicio del tratamiento. En un estudio que incluyó 250 casos de LC y LCV, $100 \%$ de los pacientes que fallecieron recibieron tratamiento después de $24 \mathrm{~h}$ de iniciado el cuadro ${ }^{7}$. Otro factor en el pronóstico es la edad del paciente, siendo más grave en niños ${ }^{18}$. El tamaño de la lesión cutánea no se correlaciona con el riesgo de desarrollar LCV ni con el pronóstico ${ }^{18}$.

El diagnóstico es clínico, se basa en la identificación del artrópodo, asociada a un cuadro clínico sugerente.
La araña es visualizada en aproximadamente $60 \%$ de los casos, pero correctamente identificada sólo en $13 \%{ }^{19}$. Existen métodos en investigación en animales para la detección de la enzima esfinomielinasa D desde $30 \mathrm{~min}$ hasta cinco días tras ocurrir la mordedura ${ }^{20}$, pero que no son de uso habitual en la práctica clínica.

El diagnóstico diferencial incluye celulitis, mordedura sobreinfectada (por otros artópodos), vasculitis y reacciones adversas a medicamentos, entre otros.

En el enfrentamiento de todo paciente con diagnóstico de loxoscelismo es imperativo descartar LCV, por lo que debe realizarse monitoreo de 24 h con exámenes. Dentro del laboratorio necesario para evaluar un compromiso visceral se incluyen hemograma, índice reticulocitario, bilirrubinemia, protrombinemia, tiempo de tromboplastina parcial activado, nitrógeno ureico en sangre, creatininemia, lactato deshidrogenasa y orina completa ${ }^{7}$.

En relación al tratamiento de esta entidad, no existen pautas establecidas y su manejo es controversial. El manejo general del loxoscelismo cutáneo incluye la aplicación de hielo local, elevación de la extremidad y curaciones simples de la herida. En los casos de LCV el manejo debe enfocarse en mantener la hidratación, monitoreo de las alteraciones hidroelectrolíticas, y manejo de la insuficiencia renal.

Se ha descrito gran variedad de tratamientos para el manejo de LC y LCV, incluyendo corticoesteroides, dapsona, antihistamínicos, suero anti-loxosceles, antimicrobianos y colchicina, entre otros ${ }^{15}$. A la fecha no existe un consenso en torno a un estándar de oro para el tratamiento de estos pacientes ${ }^{21}$, en parte porque existen pocos estudios y revisiones sistemáticas de buena calidad metodológica con respecto a las alternativas terapéuticas disponibles ${ }^{15}$.

El objetivo de este trabajo fue describir las manifestaciones clínicas, principales complicaciones, manejo terapéutico y evolución de pacientes internados por LC y LCV en el Hospital Clínico de la Pontificia Universidad Católica de Chile (HCPUC).

\section{Pacientes y Métodos}

Estudio descriptivo, retrospectivo y observacional de pacientes internados en el HCPUC entre los años 2014 a 2017 con diagnóstico de loxoscelismo y evaluados por el equipo de Dermatología.

Se obtuvieron datos de fichas clínicas, informes de biopsia, epicrisis y registro de visita dermatológica, en el que se incluyen todos los pacientes hospitalizados evaluados por el equipo de Dermatología, referidos por sus tratantes para evaluación y tratamiento de condiciones cutáneas. 
Tabla 1. Parámetros clínicos, tratamiento y resolución de 17 pacientes con loxoscelismo internados en el Hospital Clínico de la Pontificia Universidad Católica de Chile entre 2014 y 2017

\begin{tabular}{|c|c|c|c|c|c|}
\hline Características & $\mathbf{n}$ & $\%$ & Características & $\mathbf{n}$ & $\%$ \\
\hline Sexo & & & Causa de hospitalización & & \\
\hline Masculino & 9 & 53 & Sospecha de sobreinfección & 8 & 47 \\
\hline \multirow[t]{2}{*}{ Femenino } & 8 & 47 & Sospecha de compromiso visceral & 4 & 23,5 \\
\hline & & & Manejo del dolor & 2 & 11,8 \\
\hline Edad & & & Sospecha de celulitis preseptal & 1 & 5,9 \\
\hline 0 a 10 años & 0 & 0 & Sospecha de vasculitis & 1 & 5,9 \\
\hline 11 a 20 años & 1 & 5,9 & Sospecha de shock tóxico & 1 & 5,9 \\
\hline 21 a 30 años & 7 & 41 & & & \\
\hline 31 a 40 años & 4 & 23,6 & Tratamientos & & \\
\hline 41 a 50 años & 2 & 11,8 & Antihistamínicos & 17 & 100 \\
\hline 51 a 60 años & 2 & 11,8 & Dapsona & 11 & 64,7 \\
\hline \multirow[t]{2}{*}{ Mayor de 60 años } & 1 & 5,9 & Corticosteroides sistémicos & 16 & 94 \\
\hline & & & Corticosteroides tópicos & 9 & 53 \\
\hline Estación del año & & & Antimicrobianos & 17 & 100 \\
\hline Primavera & 2 & 11,8 & Colchicina & 2 & 11,8 \\
\hline Verano & 13 & 76,7 & Debridamiento quirúrgico & 1 & 5,9 \\
\hline Otoño & 2 & 11,8 & & & \\
\hline \multirow[t]{2}{*}{ Invierno } & 0 & 0 & Tratamiento de loxoscelismo cutáneo & & \\
\hline & & & Antihistamínicos & 14 & 100 \\
\hline Identificación de la araña & & & Dapsona & 10 & 71 \\
\hline Sí & 7 & 41 & Corticosteroides sistémicos & 13 & 93,3 \\
\hline \multirow[t]{2}{*}{ No } & 10 & 59 & Corticosteroides tópicos & 8 & 56,8 \\
\hline & & & Antimicrobianos & 14 & 100 \\
\hline \multicolumn{3}{|l|}{ Tipo } & Colchicina & 1 & 7,1 \\
\hline Cutáneo & 14 & 82,3 & Drenaje quirúrgico de absceso secundario & 1 & 7,1 \\
\hline Cutáneo-edematoso & 1 & 5,9 & & & \\
\hline Cutáneo-visceral & 2 & 11,8 & Tratamiento de loxoscelismo cutáneo-visceral & & \\
\hline \multirow{2}{*}{\multicolumn{3}{|c|}{ Localización }} & Antihistamínicos & 2 & 100 \\
\hline & & & Dapsona & 1 & 50 \\
\hline Cara & 1 & $\begin{array}{r}5,9 \\
520\end{array}$ & Corticosteroides sistémicos & 2 & 100 \\
\hline EESS & 9 & 52,9 & Corticosteroides tópicos & 1 & 50 \\
\hline EEII & 4 & 23,5 & Antimicrobianos & 2 & 100 \\
\hline Tronco & 2 & 11,7 & Colchicina & 1 & 50 \\
\hline Glúteos & 1 & 5,9 & Debridamiento quirúrgico & 1 & 50 \\
\hline \multicolumn{3}{|l|}{ Clínica } & Evolución & & \\
\hline Dolor & 17 & 100 & Resolución total & 10 & 59 \\
\hline Eritema & 17 & 100 & Cicatriz atrófica & 3 & 17,7 \\
\hline Edema & 15 & 88,2 & Hiperpigmentación post inflamatoria & 1 & 5,9 \\
\hline Necrosis & 10 & 59 & Escarectomía & 2 & 11,8 \\
\hline Fiebre & 6 & 35 & Necrosis digital & 1 & 5,9 \\
\hline Rash no atribuible a fármacos & 6 & $\begin{array}{l}35 \\
35\end{array}$ & Muerte & 0 & 0 \\
\hline Sospecha de sobreinfección & 6 & 35 & & & \\
\hline $\begin{array}{l}\text { Ampolla } \\
\text { Hemólisis_Hematuria }\end{array}$ & 5 & 29 & & & \\
\hline Hemólisis-Hematuria & 2 & 11,8 & & & \\
\hline
\end{tabular}

\section{Resultados}

Se registraron 17 casos de pacientes hospitalizados con diagnóstico de loxoscelismo (Tabla 1), de los cuales $82,3 \%$ (n: 14) correspondían a LC (Figura 1), 5,9\% (n: 1) a LCPE (de localización peri ocular) y $11,8 \%$ (n: 2) a LCV (Figura 2).

La edad promedio fue de 36 años con un rango entre 11 a 77 años; $47 \%$ fueron de sexo femenino.
El $88,3 \%$ se presentó entre los meses de diciembre y abril, 11,7\% ( 2 casos) se presentaron en el mes de septiembre.

El 41\% de los casos identificó a una araña como la responsable de la mordedura, pero sólo en un caso pudo ser capturada para identificación específica como L. laeta.

Dos pacientes hospitalizadas por loxoscelismo estaban embarazadas; ambas fueron manejadas con antibioticoterapia: ceftriaxona y luego clindamicina, clorfenamina y corticosteroides tópicos, con resolución completa de la lesión al mes de evolución. 


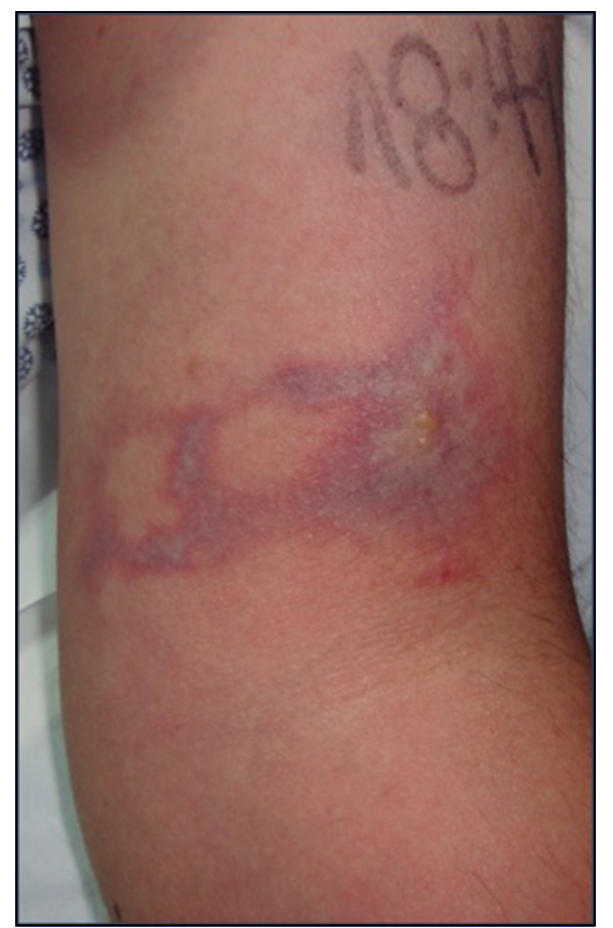

Figura 1. Loxoscelismo cutáneo necrótico.

En promedio, transcurrieron 2,5 días entre la mordedura y la hospitalización, con un rango entre 1 y 5 días.

La frecuencia de localización de la mordedura en orden decreciente fue: extremidades superiores en 52,9\%, extremidades inferiores en $23,5 \%$, tronco en $11,7 \%$, cara en $5,9 \%$ y glúteo en $5,9 \%$ (Figura 3 ).

Con respecto a las características clínicas, el dolor y eritema se presentaron en $100 \%$ de los casos, edema en $88,2 \%$, necrosis en $59 \%$, fiebre en $35 \%$ (incluyendo casos de LC y LCV), rash maculopapular no atribuible a fármacos en 35\% (Figura 3A y 3B) y sospecha de sobreinfección al ingreso en $35 \%$. Veintinueve por ciento de los pacientes evolucionó con ampolla en la lesión cutánea. En promedio las lesiones tuvieron un diámetro de 13,3 $\mathrm{cm}$ con un rango entre 4 y $30 \mathrm{~cm}$ (tamaño registrado en sólo 10 casos).

La principal causa de hospitalización fue sospecha de celulitis o sobreinfección (47\%), seguida de sospecha de LCV en $23,5 \%$. Otras razones fueron sospecha de vasculitis y manejo del dolor. El promedio de días de hospitalización fue de 5,8 días (rango entre 1 y 9 días).

Con respecto al manejo terapéutico, todos los casos fueron manejados con antibioterapia intravenosa, siendo en $88 \%$ cefalosporinas $(80 \%$ cefazolina o cefadroxilo, $20 \%$ ceftriaxona). En un caso se debió cambiar a clindamicina por aparición de rash. Otros antimicrobianos administrados incluyeron cefadroxilo, penicilinas, clindamicina y doxiciclina.

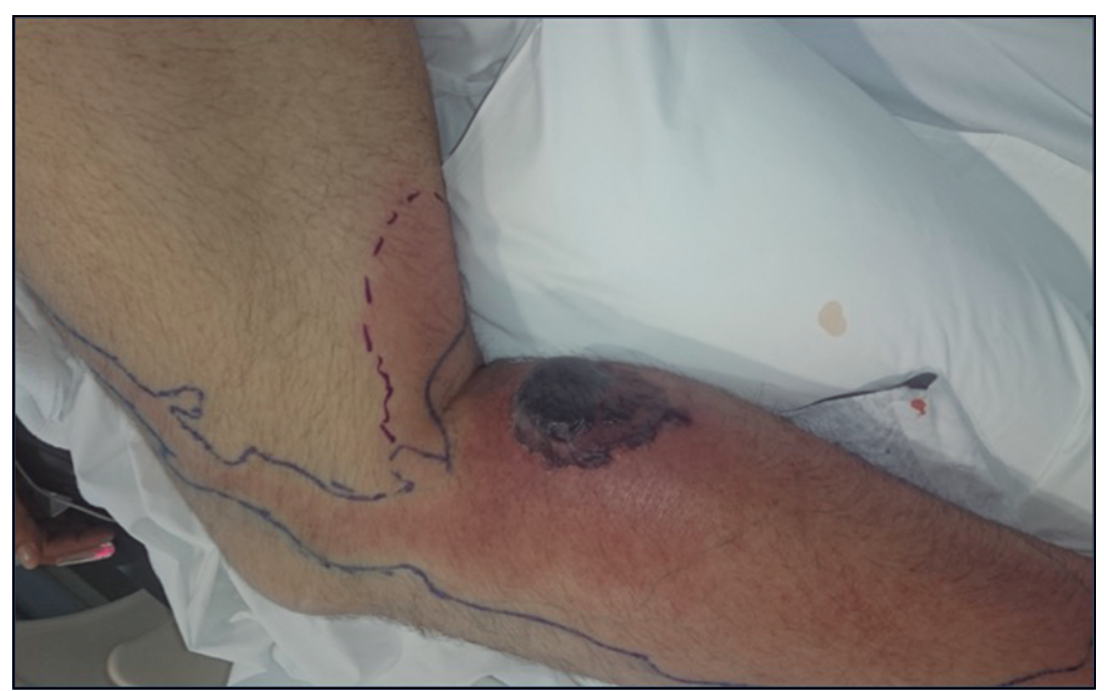

Figura 2. Loxoscelismo cutáneo-visceral.

Un paciente recibió inmunoglobulina intravenosa a su ingreso por sospecha de shock tóxico, pero se suspendió tratamiento una vez establecido el diagnóstico. El 100\% recibió antihistamínicos (clorfenamina) en algún momento durante la evolución del cuadro.

Noventa y cuatro por ciento recibió corticosteroides sistémicos; aproximadamente la mitad comenzó con hidrocortisona para luego recibir prednisona en dosis de 0,5 a $1 \mathrm{mg} / \mathrm{kg} /$ día, y la otra mitad recibió prednisona desde el inicio. La terapia con corticosteroides se extendió en promedio por siete días en los casos de LC y hasta un mes en los LCV. El 53\% recibió corticosteroides tópicos, siendo la mayoría clobetasol.

En 64,7\% de los casos se agregó dapsona (50-150 mg al día por 4 a 6 semanas, una vez descartado el compromiso visceral), debiendo suspenderse en un paciente por anemia hemolítica secundaria (aún cuando la actividad enzimática de glucosa-6-fosfato deshidrogenasa se encontraba normal previo al inicio de fármaco) y en otro por aparición de rash maculopapular. En ambos casos se indicó colchicina $0,5 \mathrm{mg}$ cada $12 \mathrm{~h}$; no obstante, ambos evolucionaron desfavorablemente requiriendo escarectomía posterior.

Un paciente con LC presentó un absceso sobre la úlcera necrótica que requirió drenaje quirúrgico, obteniendo cultivo positivo para Staphylococcus aureus multisensible.

El $71,4 \%$ de los pacientes con LC fue manejado con corticosteroides y dapsona simultáneamente. De ellos, $50 \%$ presentó resolución completa de la lesión al mes de tratamiento. Cuatro pacientes recibieron terapia esteroidal sistémica sin dapsona, de quienes $75 \%$ presentó resolución completa al mes de evolución. No se registraron pacientes que recibieran dapsona sin haber recibido tratamiento con corticosteroides. 


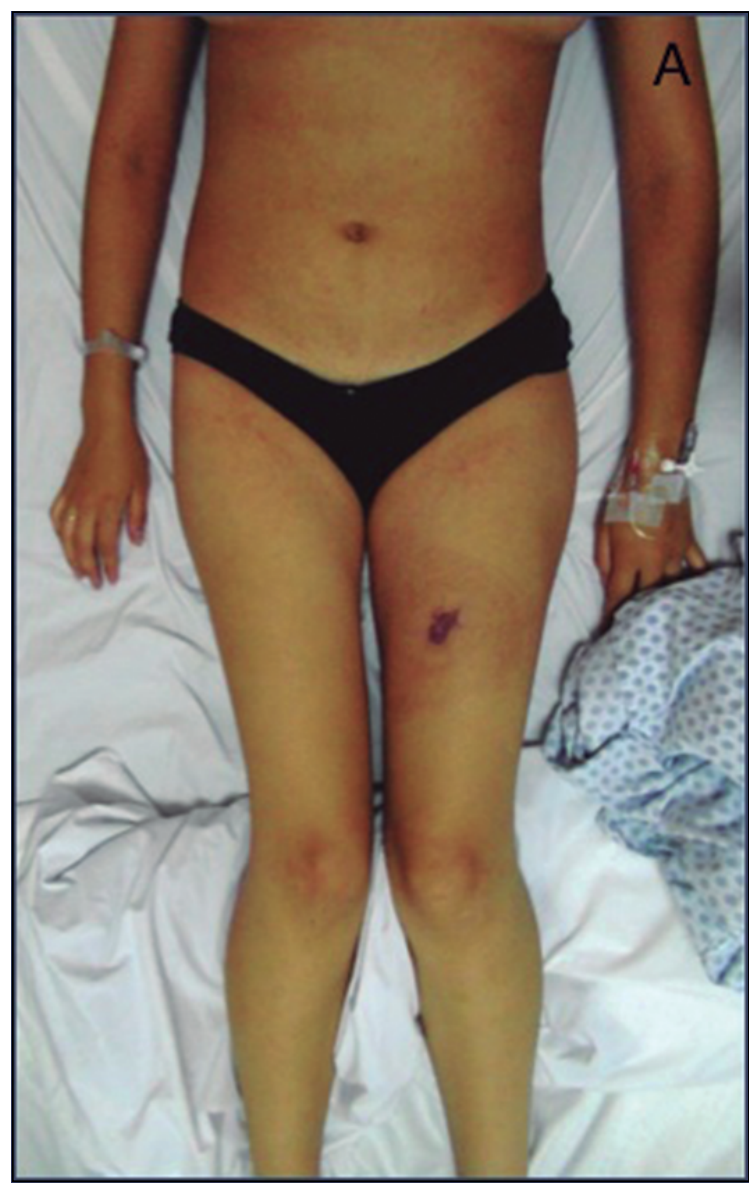

Figura 3A. Loxoscelismo cutáneo necrótico asociado a rash morbiliforme no atribuible a fármacos.

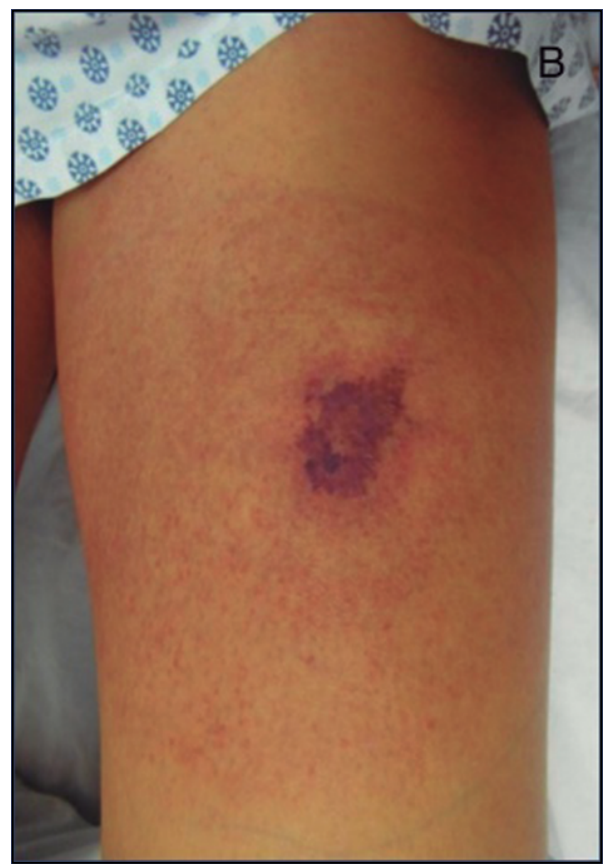

Los dos casos de LCV se presentaron con hemólisis, hematuria y elevación de transaminasas hepáticas.

El primer caso consultó con $48 \mathrm{~h}$ de evolución de fiebre, compromiso del estado general, y lesión indurada violácea dolorosa de $12 \mathrm{~cm}$ en el muslo. Dentro de sus exámenes de ingreso destacó hemograma normal, creatininemia de $1,1 \mathrm{mg} / \mathrm{dl}$, pruebas de función hepática elevadas (SGOT 59 U/1, SGPT $93 \mathrm{U} / 1$, GGT $82 \mathrm{U} / 1$, bilirribinemia total normal), orina con hematuria (32 eritrocitos por campo) y lactato deshidrogenasa levemente elevada. Se inició manejo de soporte y tratamiento con cefazolina, prednisona $40 \mathrm{mg}$ al día, fluticasona tópica y clorfenamina. Tras confirmar una actividad normal de la enzima glucosa-6-fosfato deshidrogenasa, se agregó tratamiento con dapsona. Luego de cuatro días, el paciente fue dado de alta continuando con cefadroxilo durante una semana, dapsona y prednisona. En el control ambulatorio se disminuyó progresivamente la dosis de prednisona -fue suspendida a los 18 días- y completó tratamiento con dapsona por un mes $(150 \mathrm{mg} /$ día por 21 días y $100 \mathrm{mg} /$ día por siete días) con excelente respuesta y sin cicatriz residual.

El segundo caso de LCV consultó en nuestro hospital cinco días después de ocurrida la mordedura, con anemia (hematocrito $30,2 \%$, índice reticulocitario 1,6 ), plaquetopenia, elevación de LDH, transaminasas hepáticas y de bilirrubinemia (predominio directo); y una extensa úlcera necrótica (con placa livedoide de $30 \mathrm{~cm}$ de diámetro mayor), requiriendo a su ingreso desbridamiento quirúrgico y aplicación de hielo local. Se inició manejo de soporte y tratamiento con cefazolina, hidrocortisona $100 \mathrm{mg}$ cada 8 h, y clorfenamina. Luego de seis días, se decidió continuar manejo ambulatorio con prednisona $60 \mathrm{mg}$ por cinco días, doxiciclina $100 \mathrm{mg}$ al día y betametasona con ácido fusídico tópico por un mes, tras lo cual requirió escarectomía.

Ningún caso de LCV presentó otras complicaciones médicas, normalizando los exámenes de laboratorio durante el primer mes de evolución con los tratamientos descritos.

En relación a la evolución clínica de todos los casos, $59 \%$ no desarrolló úlcera residual, cursaron hacia la resolución de las lesiones al mes de tratamiento y la aparición de cicatriz residual leve o hiperpigmentación post-inflamatoria. Dos casos requirieron escarectomía para tratar la zona de necrosis y el paciente que tuvo la mordedura de la araña en el dedo requirió operación por necrosis digital.

\section{Discusión}

El loxoscelismo es una patología relativamente frecuente en nuestro medio, con un amplio espectro de
Figura 3B. Aumento de la imagen en el sitio de la mordedura. 
presentaciones clínicas y diagnósticos diferenciales, y con potenciales complicaciones que pueden requerir manejo médico intensivo, intervención quirúrgica, o incluso provocar la muerte.

Los resultados obtenidos en este estudio representan una primera aproximación a la caracterización clínicoepidemiológica de los pacientes con loxoscelismo manejados en ambiente hospitalario. Si bien el grupo estudiado no constituye una muestra representativa de la población chilena en términos estrictamente estadísticos, sí puede ser considerado como una referencia inicial que permita caracterizar la clínica, manejo y evolución de esta enfermedad en pacientes que requieren manejo hospitalario.

La presentación clínica de los casos en nuestro hospital responde al patrón epidemiológico descrito previamente en la literatura médica, mostrando un franco predominio de LC frente a LCV. La edad de presentación más frecuente fue entre la tercera y cuarta década de la vida, con predominio en pacientes de sexo masculino, siendo las estaciones de verano y otoño las épocas del año más asociadas a la mordedura.

En nuestra serie de pacientes, la identificación de la araña se realizó en menos de la mitad de los casos, por lo que se debe tener un alto nivel de sospecha frente a un cuadro clínico sugerente y no debiera ser descartado por la ausencia de este elemento en la anamnesis.

Con respecto a las características clínicas, la localización más frecuente fue en las extremidades, siendo el dolor y eritema la forma de presentación inicial de todos los pacientes; la necrosis cutánea sólo estaba en la mitad de los casos. Este hallazgo es de vital importancia en la evaluación precoz de los casos de loxoscelismo, enfatizando que la clásica placa livedoide es poco frecuente como presentación inicial del cuadro y la sospecha ante un dolor desproporcionado en relación a la lesión cutánea, junto a una historia clínica compatible, debería primar para establecer el diagnóstico.

La fiebre se presentó en un tercio de los pacientes, independientemente del subtipo de loxoscelismo, lo que sugiere que no debe ser considerado como un factor clave de compromiso visceral.

En relación al enfrentamiento terapéutico, existen distintas alternativas de tratamiento disponibles, pero escasa evidencia en la literatura médica de su real beneficio en este cuadro. Dentro de éstos, dapsona crea particular interés, y si bien no existe evidencia sólida que respalde su efectividad, su uso parece adecuado particularmente en casos de consulta precoz, cuando la necrosis extensa aún no se ha establecido ${ }^{15}$.

El uso de dapsona como tratamiento para el loxoscelismo fue introducido por King y Rees en $1983^{22}$, y desde entonces se han reportado diferentes series de casos que avalan su uso. Se ha descrito que con el uso de dapsona las lesiones se circunscriben más rápido, con disminución del dolor, inflamación y necrosis y con cicatricación acelerada de la herida ${ }^{23}$. Se justificaría su uso en pacientes con cuadros graves, con necrosis, bulas, hemorragia o exudado purulento, en cuadros leves podría no justificarse dado sus potenciales efectos adversos ${ }^{15}$.

En Chile, el uso de dapsona para LC ha sido reportado previamente con resultados favorables ${ }^{23}$. En 2009 Manríquez y Silva publicaron una revisión sistemática de 10 estudios de tratamiento en pacientes con LC; entre ellos, cuatro comparaban dapsona con otro tipo de tratamiento ${ }^{15}$. Si bien los estudios son de pobre calidad metodológica, permitieron concluir que no habría diferencia entre el uso de dapsona en dosis de $100 \mathrm{mg}$ al día en combinación con suero anti-Loxosceles reclusa obtenido de conejos en dosis de 0,2 $\mathrm{ml}$ intra-lesional, y el uso de cada uno de ellos por separado en términos de acortar el tiempo hasta la curación de la herida cutánea ${ }^{24}$. En casos de LC con evolución entre $24 \mathrm{~h}$ y cinco días, el uso de dapsona fue superior al uso de clorfenamina en lograr evolución favorable de la herida cutánea ${ }^{25}$. Dapsona también sería efectiva en el tratamiento iniciado entre los cinco y 20 días ${ }^{26}$. Además, el tratamiento con dapsona, seguido de tratamiento quirúrgico según fuese la evolución clínica, sería más efectivo que el tratamiento quirúrgico precoz en evitar el retraso de la cicatrización o cicatrización anómala ${ }^{27}$. En pacientes pediátricos con LC, el tratamiento con dapsona $1 \mathrm{mg} / \mathrm{kg}$ de peso al día por siete a 14 días asociado a cloxacilina (si hubiere signos de infección), sería efectivo en lograr la mejoría completa de las lesiones cutáneas $^{28}$. Sin embargo, mediante análisis multivariado, se ha demostrado que el uso de dapsona y corticosteroides sistémicos se asociaría a un retraso en la curación de la herida cutánea ${ }^{29}$.

Dentro de los efectos adversos reportados al uso de dapsona se encuentran molestias gastrointestinales, rash cutáneo $^{26}$, ictericia colestásica, hepatitis, leucopenia, meta-hemoglobinemia, neuropatía periférica ${ }^{30,31}$, fotosensibilidad $^{32}$, síndrome de hipersensibilidad a la dapsona y neumonía eosinofílica ${ }^{33}$. Además, existe el riesgo de desarrollar anemia hemolítica ${ }^{34}$, especialmente en sujetos con déficit de la enzima glucosa-6-fosfato deshidrogenasa. Esta situación debe ser considerada particularmente en pacientes con loxoscelismo en tratamiento con este fármaco, ya que la anemia hemolítica puede ser difícil de diferenciar como efecto de la dapsona frente el potencial compromiso sistémico del veneno de la araña. Así, su uso debiera iniciarse una vez descartado el compromiso sistémico $^{15}$. Por los posibles efectos adversos descritos se sugiere control con exámenes periódicos durante los primeros dos meses de tratamiento ${ }^{3}$.

Clorfenamina es un fármaco que se usa en todos los pacientes con loxoscelismo en el HCPUC, y aunque su evidencia muestra que es menos efectiva que otras inter- 
venciones, es una alternativa con bajo riesgo de efectos adversos y que ha mostrado efectividad en disminuir el dolor y edema en pacientes con loxoscelismo ${ }^{35}$.

El uso de corticosteroides es discutido ${ }^{15} \mathrm{y}$, si bien no existe evidencia que avale su uso, la guía clínica del MINSAL de 2016 recomienda su administración sistémica en casos de $\mathrm{LCV}^{18}$, ya que su efecto inmunosupresor podría limitar la gravedad del cuadro $^{36}$. Se recomienda la vía parenteral, ya que en autopsias de pacientes fallecidos por esta patología se ha observado edema de la mucosa intestinal que dificultaría la efectividad de la administración oral ${ }^{8}$. Se ha reportado un caso de LCV con síndrome hemofagocítico secundario que presentó respuesta completa al tratamiento con dexametasona intravenosa ${ }^{37}$. En casos de LC el uso de corticosteroides sistémicos es controversial, ya que fisiopatológicamente tendría un rol como anti-inflamatorio e inmunosupresor, pero un estudio asoció su uso a retraso en la curación de la herida cutánea ${ }^{29}$. El uso de corticosteroides tópicos no tiene respaldo en evidencia científica; sin embargo, es una práctica clínica habitual en pacientes con loxoscelismo.

Considerando nuestra experiencia, el uso de corticosteroides sistémicos en dosis de 0,5 a $1 \mathrm{mg} / \mathrm{kg} /$ día por un período limitado, sí sería beneficioso para detener la cascada proinflamatoria asociada a la mordedura por loxosceles. Esta medida sería particularmente útil en pacientes con LC complicado, cuadros aquellos que puedan evolucionar con escaras o gran cicatriz residual, como son aquellas presentaciones con necrosis, gran extensión, localización compleja (periocular), ampolla o con dolor importante.

Otra herramienta terapéutica es el uso de antimicrobianos. Se ha descrito su uso profiláctico para prevenir infecciones secundarias por Streptococcus pyogenes y Staphylococcus aureus ${ }^{15}$. En un estudio en Antofagasta (Chile) se observó presencia de Clostridium perfringens en colmillos de $15,7 \%$ de especímenes de $L$. laeta recolectados, lo que en asociación al veneno de la araña produce exacerbación del daño dermonecrótico ${ }^{38}$.

Por otra parte, se ha observado en modelos in vitro y en animales con LC inducido por veneno de L. laeta un aumento de la expresión o actividad de metaloproteinasas 2,7 y 9, con asociación directa en el daño dermonecrótico $^{10}$. En modelos in vitro el uso de tetraciclina logró prevenir la muerte celular secundaria a este veneno y la administración tópica de tetraciclina dos veces al día por $48 \mathrm{~h}$ disminuyó la lesión dermonecrótica en modelos de conejo, asociada a disminución de infiltración de neutrófilos ${ }^{10}$. El uso de tetraciclina también ha demostrado disminución de dermonecrosis en modelos de LC en conejos secundario a $L$. intermedia, asociado a disminución de actividad de metaloproteinasas 2 y $9^{39}$. De esta manera, antimicrobianos de la familia de las tetraciclinas podrían tener un rol en el manejo de pacientes con loxoscelismo debido a su actividad antiinflamatoria.

Si bien uno de los principales tratamientos conocidos por la población general es el suero anti-loxosceles, se ha comprobado que éste inhibe la formación de lesiones cutáneas necróticas sólo al ser administrado hasta una hora post mordedura ${ }^{34}$. Además, no inhibe la hemólisis inducida por el veneno ${ }^{34}$. Por lo tanto, dado la poca evidencia clínica del beneficio que pudiera aportar el suero antiloxosceles, actualmente no se recomienda su uso ${ }^{18} \mathrm{y}$ no se utiliza en nuestro centro.

Existen trabajos prometedores con el uso de anticuerpos monoclonales humanizados contra la esfingomielinasa $\mathrm{D}^{40}$, aún no disponibles en la práctica clínica.

\section{Conclusión}

Se describen 17 casos de loxoscelismo de manejo hospitalario, de los que 11,8\% tuvieron una presentación clínica compatible con LCV. La mayoría de los casos, con LC, fue manejada con medidas de soporte, antimicrobianos, corticosteroides sistémicos, antihistamínicos y dapsona, con excelente respuesta, rápida resolución del cuadro y escasa cicatriz residual en la placa livedoide. Considerando nuestros hallazgos proponemos el siguiente algoritmo para el manejo de los cuadros de loxoscelismo (Figura 4).

La falta de publicaciones de series clínicas y de evidencia de buena calidad sobre el enfrentamiento terapéutico del loxoscelismo pone de manifiesto la necesidad de realizar nuevos estudios clínicos dirigidos a mejorar el conocimiento respecto a esta enfermedad y a definir las mejores terapias para evitar sus co-morbilidades.

\section{Resumen}

Introducción: El loxoscelismo es una patología frecuente en nuestro medio con un amplio espectro de presentaciones y diagnósticos diferenciales, con complicaciones potencialmente graves, e incluso con riesgo de muerte. A la fecha no existe un tratamiento estándar para estos pacientes. Objetivo: Describir las manifestaciones clínicas, principales complicaciones, manejo terapéutico y evolución de pacientes internados por loxoscelismo en un hospital terciario en Chile. Pacientes y Método: Se analizaron todos los pacientes consultantes e internados por loxoscelismo en el Hospital Clínico de la Pontificia Universidad Católica de Chile entre los años 2014 y 2017, evaluados en interconsulta por Dermatología. Revisión de los registros clínicos incluyendo semiología, imágenes, informes de laboratorio y tratamientos efectuados. Resultados: Se registraron 17 casos de loxoscelismo 


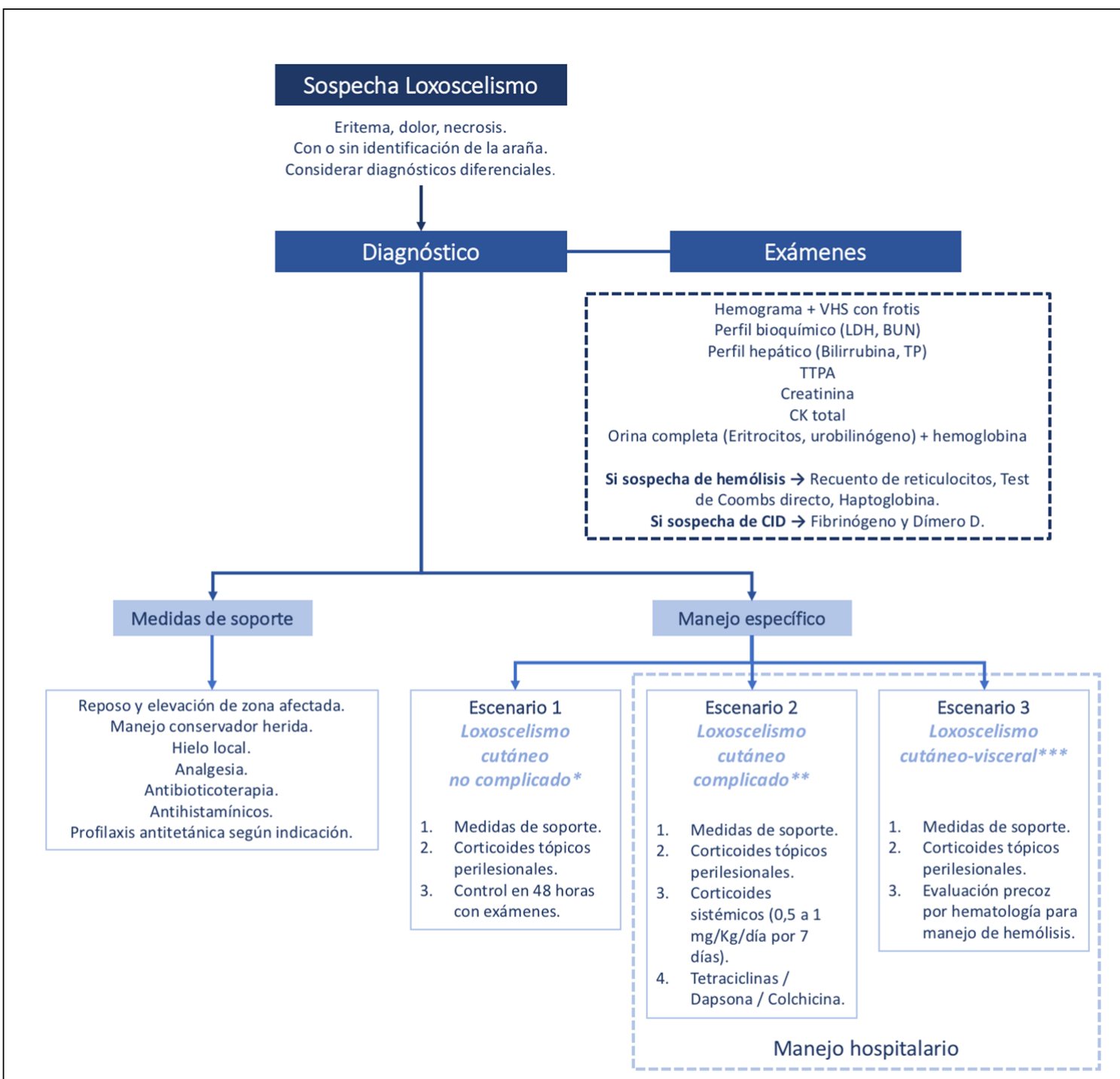

Placa livedoide estable $\rightarrow$ Evaluación posterior con Cirugía Plástica

*LC no complicado: extensión limitada, sin necrosis, localización no compleja, sin signos de sobreinfección bacteriana, dolor moderado ** LC complicado: compromiso extenso, con necrosis, localización compleja (cara), sobreinfección bacteriana o dolor difícil de manejar ***LCV: Alteraciones de laboratorio sugerentes de hemólisis

de manejo hospitalario, cuya presentación responde al patrón epidemiológico nacional. La mayoría de los casos fue manejada con antimicrobianos, corticosteroides sistémicos, antihistamínicos y dapsona. De ellos, 11,8\% correspondieron a loxoscelismo cutáneo visceral, manejados exitosamente con medidas de soporte, corticosteroides sistémicos y antihistamínicos. El 59\% presentó resolución de las lesiones al mes de tratamiento, con cicatriz residual leve o hiperpigmentación postinflamatoria, sin mortalidad en nuestra serie. Discusión: La mayoría de los casos de loxoscelismo cutáneo presentó excelente respuesta y rápida resolución del cuadro tras el tratamiento asociado de corticosteroides sistémicos, antimicrobianos y dapsona, sugiriendo que el uso de estas terapias podría detener la progresión de la necrosis cutánea y prevenir las complicaciones asociadas al loxoscelismo.
Figura 4. Propuesta de algoritmo para el manejo de pacientes con cuadros de loxoscelismo. 


\section{Referencias bibliográficas}

1.- Isbister G K, Fan H W. Spider bite. Lancet 2011; 378: 2039-47. doi: 10.1016/S01406736(10)62230-1.

2.- Ríos J C, Pérez M, Sánchez P, Bettini M, Mieres J J , Paris E. Prevalence and epidemiology of Loxosceles laeta bite. Analysis of consultations to a poison control center. Rev Med Chile 2007; 135: 1160-5. DOI: /S0034-98872007000900010.

3.- Instituto de Salud Pública de Chile. Araña de los Rincones: Consulta - Respuesta. Disponible: http:// www.ispch.cl/oirs/SIAC_2007/siac. php?page $=$ A\&respuesta $=$ ARA $\%$ D1A $\% 20$ DE\%20LOS\%20RINCONES\&cod=9. (Accedido el 25 de junio de 2017).

4.- Araya J E. Caracterización a nivel molecular del veneno de Loxosceles laeta (araña de rincón). Obtención de un antídoto específico y elaboración de un kit diagnóstico para detección temprana de la mordedura. Informe final proyecto FONDEF. Disponible: http://146.83.150.183/bitstream/ handle/10533/88619/pdf_informe_final $\% 20$ D04I1247.pdf?sequence=1 (Accedido el 15 de junio de 2017).

5.- Parra D, Torres M, Morillas J, Espinoza P. Loxosceles laeta, identificación y una mirada bajo microscopía de barrido. Parasitol Latinoam 2002; 57: 75-8. https://scielo.conicyt.cl/pdf/ parasitol/v57n1-2/art19.pdf.

6.- Sezerino U M, Zannin M, Coelho L K, Gonçalves Júnior J, Grando M, Mattosinho S $\mathrm{G}$, et al. A clinical and epidemiological study of Loxosceles spider envenoming in Santa Catarina, Brazil. Trans R Soc Trop Med Hyg 1998; 92: 546-8.

7.- Schenone H. Cuadros tóxicos producidos por mordeduras de araña en Chile: latrodectismo y loxoscelismo. Rev Med Chile 2003; 131: 437-44. https://scielo. conicyt.cl/scielo.php?script=sci_arttext\&pid $=$ S0034-98872003000400013.

8.- Schenone H. A propósito del loxoscelismo en Chile. Rev Med Chile 2004; 132: 121-2. http://dx.doi.org/10.4067/S003498872004000100018.

9.- Saraco S, De Roodt A. Envenenamiento por animales ponzoñosos. Loxoscelismo. ATA 2005; 67: 45-9.

10.- Corrêa M A, Okamoto C K, Gonçalves-deAndrade R M, van den Berg C W, Tambourgi D V. Sphingomyelinase D from Loxosceles laeta venom induces the expression of MMP7 in human keratinocytes: contribution to dermonecrosis. PLoS One 2016; 11: e0153090. doi: 10.1371/journal.pone.0153090.

11.- Tambourgi D V, Gonçalves-de-Andrade R M, van den Berg C W. Loxoscelism: From basic research to the proposal of new therapies. Toxicon 2010; 56: 1113-9. doi: 10.1016/j. toxicon.2010.01.021.

12.- la Barra P, Vial V, Labraña Y, Álvarez A M, Seguel H. Cutaneous loxoscelism mainly edematous: a case report. Rev Chilena Infectol 2015; 32: 467-71. doi: 10.4067/S071610182015000500017.

13.- Cesaroni E, González S. Loxoscelismo cutáneo: a propósito de 6 casos. Arch Argent Dermatol 2006; 66: 71-77. http://www.archivosdermato. org.ar/Uploads/71CesaroniLoxoscelismo $\% 20$ cut $\% \mathrm{C} 3 \% \mathrm{~A} 1$ neo.pdf.

14.- Valverde López J. Aspectos clínicos y epidemiológicos del loxoscelismo, Hospital Regional Docente de Trujillo, enero de 2001 a noviembre de 2003. Folia Dermatol Perú 2003; 14: 15-9.

15.- Manríquez J J , Silva S. Cutaneous and visceral loxoscelism: a systematic review. Rev Chilena Infectol 2009; 26: 420-32. http://dx.doi. org/10.4067/S0716-10182015000300014.

16.- Ríos J C, Paris E, Apt W, Ristori L, Ramírez G. Guía clínica para el manejo de la mordedura de araña de rincón. Loxosceles laeta. Ministerio de Salud. 2004. http://infomedic.cl/data/ documents/Loxosceles.pdf.

17.- Tambourgi D, Paixao-Cavalcante D, Goncalves R, Fernándes-Pedrosa M F, Morgan P, Van De Berg C. Loxosceles sphingomyelinase induces complement- dependent dermonecrosis, neutrophil infiltration, and endogenous gelatinase expression. J Invest Dermatol 2005; 124: 72531. DOI: 10.1111/j.0022-202X.2005.23654.X

18.- Apt W, Denegri M, Jofré L, Noemí I, Tassara R, Torres M, et al. Guía clínica para el Manejo de Mordedura de Araña de los Rincones Loxosceles laeta. Ministerio de Salud, Chile, 2016. http://www.minsal.cl/wp-content/ uploads/2016/11/LOXOSCELES-FINAL.pdf.

19.- Pichardo-Rodríguez R. Possible clinicalepidemiological criteria for the diagnosis of loxoscelism cutaneous and visceral cutaneous. Rev Chilena Infectol 2013; 30: 453. DOI: 10.4067/S0716-10182013000400017.

20.- Pichardo-Rodríguez R. A possible gold standard for the diagnosis of loxoscelism and a solution for research. Rev Chilena Infectol 2014; 31: 353. DOI:10.4067/S071610182014000300018.

21.- Swanson D L, Vetter R S. Loxoscelism. Clin Dermatol 2006; 24: 213-21. DOI: 10.1016/j. clindermatol.2005.11.006.

22.- King L E, Rees R S. Dapsone treatment of a brown recluse bite. JAMA 1983; 250: 648.
23.- Benavides M I, Moncada X. The treatment of cutaneous loxoscelism with dapsone. Rev Med Chile 1990; 118: 1247-50.

24.- Rees R, Campbell D, Rieger E, King L E. The diagnosis and treatment of brown recluse spider bites. Ann Emerg Med 1987; 16: 945-9.

25.- Maguiña Vargas C, Gotuzzo Herencia E, Álvarez H. Nuevos esquemas terapéuticos en loxoscelismo cutáneo en Lima, Perú. Folia Dermatol Perú 1997; 8: 23-30.

26.- Maguiña Vargas C. La dapsona (DDS) en el loxoscelismo cutáneo. Diagnóstico (Perú) 1987; 20: 58-64.

27.- Rees R S, Altenbern D P, Lynch J B , King L E. Brown recluse spider bites. A comparison of early surgical excision versus dapsone and delayed surgical excision. Ann Surg 1985; 202 : 659-63.

28.- Escalante Galindo P. Loxoscelismo local dermonecrótico en niños mordidos por la araña Loxosceles reclusa (araña "violinista"). Gac Méd Méx 1999; 135: 423-6.

29.- Mold J W, Thompson D M. Management of brown recluse spider bites in primary care. $\mathrm{J}$ Am Board Fam Pract 2004; 17: 347-52.

30.- Iserson K V. Methemoglobinemia from dapsone therapy for a suspected brown spider bite. $\mathrm{J}$ Emerg Med 1985; 3: 285-8.

31.- Pfeiffer C, Wozel G. Dapsone and sulfones in dermatology: overview and update. J Am Acad Dermatol 2003; 48: 308-9. DOI: 10.1067/ mjd.2001.114733.

32.- Stockel S, Meurer M, Wozel G. Dapsoneinduced photodermatitis in a patient with linear IgA dermatosis. Eur J Dermatol 2001; 11: 50-3.

33.- Jaffuel D, Lebel B, Hillaire-Buys D, Pene J, Godard P, Michel FB, et al. Eosinophilic pneumonia induced by dapsone. BMJ 1998; 317: 181 .

34.- Bravo M, Oviedo I, Farías P, Schenone H. Study of anti-loxosceles serum action on hemolytic and ulcero-necrotic cutaneous effects of Loxosceles laeta venom. Rev Med Chile 1994; 122: 625-9.

35.- Schenone H. Estudio de 27 casos de loxoscelismo. Bol Chil Parasitol 1959; 14 : 7-13.

36.- Pauli I, Puka J, Gubert I C, Minozzo J C. The efficacy of antivenom in loxoscelism treatment. Toxicon 2006; 48: 123-37. DOI: 10.1016/j. toxicon.2006.05.005.

37.- Dandoy C, Grimley M. Secondary hemophagocytic lymphohistiocytosis (HLH) from a presumed brown recluse spider bite. J Clin Immunol. 2014; 34(5): 544-7. doi: 10.1007/s10875-014-0036-1.

38.- Catalán A, Espoz M C, Cortés W, Sagua H, González J, Araya J E. 
Tetracycline and penicillin resistant Clostridium perfringens isolated from the fangs and venom glands of Loxosceles laeta: its implications in loxoscelism treatment. Toxicon 2010; 56: 890-6. doi: 10.1016/j. toxicon.2010.06.012.

39.- Paixão-Cavalcante $\mathrm{D}$, van den Berg C W,
Fernandes-Pedrosa M F, Gonçalves-deAndrade R M, Tam- bourgi D V. Role of matrix metalloproteinases in $\mathrm{HaCaT}$ keratinocytes apoptosis induced by Loxosceles venom sphingomyelinase D. J Invest Dermatol 2006; 126: 61-8. http://dx.doi.org/10.1038/ sj.jid.5700049.
40.- Dias-Lopes C, Felicori L, Rubrecht L, Cobo S, Molina I, Nguyenb C, et al. Generation and molecular characterization of a monoclonal antibody reactive with conserved epitope in sphingomyelinases D from Loxosceles spider venoms. Vaccine 2014; 32: 2086-92. doi: 10.1016/j.vaccine.2014.02.012. 